\title{
NITRATO E NITRITO EM LEITE PRODUZIDO EM SISTEMAS CONVENCIONAL E ORGÂNICO'
}

\author{
Joice S. dos SANTOS ; Lígia BECK ; Melissa WALTER ; Marines SOBCZAK ; Clair J. OLIVO ; Ijoni \\ COSTABEBER ; Tatiana EMANUELLI
}

\section{RESUMO}

Nitratos e nitritos podem estar presentes naturalmente em diversos alimentos, entre eles o leite. Devido a sua toxicidade, são estabelecidos limites para a presença destes compostos nos alimentos. Assim, investigou-se os niveis de nitratos e nitritos em amostras de leite in natura, produzido por vacas submetidas aos sistemas de manejo convencional e orgânico. As amostras foram coletadas em propriedades da Região da Depressão Central do Rio Grande do Sul, Brasil. Os teores médios de nitrato e nitrito encontrados nas amostras $(\mathrm{n}=45)$ foram de $6,65 \pm 0,84 \mathrm{mg} / \mathrm{L}$ e $1,76 \pm 0,17 \mathrm{mg} / \mathrm{L}$, respectivamente. O leite produzido no sistema orgânico apresentou $7,08 \mathrm{mg} / \mathrm{L}$ de nitrato e $1,61 \mathrm{mg} / \mathrm{L}$ de nitrito, enquanto o produzido no sistema convencional apresentou 6,36 e $1,87 \mathrm{mg} / \mathrm{L}$, respectivamente. Não foi observada diferença significativa entre os teores de nitrato e nitrito encontrados nas amostras de leite em função do sistema de produção avaliado, época de coleta, turno de ordenha ou local de origem, isto é, propriedade produtora.

Palavras-chave: leite in natura; sistema de produção convencional; sistema de produção orgânico; contaminantes.

\section{SUMMARY}

NITRATE AND NITRITE IN MILK PRODUCED BY CONVENTIONAL AND ORGANIC SYSTEMS. Nitrates and nitrites can be naturally found in many foods, including milk. Due to their toxicity there is a maximum level allowed for these compounds in foods. In the present study, nitrate and nitrite levels were evaluated in raw milk samples produced in conventional and organic systems. Samples were collected from farms of the Depressão Central of Rio Grande do Sul, Brazil. The average levels of nitrate and nitrite in samples $(n=45)$ were $6.65 \pm 0.84 \mathrm{mg} / \mathrm{L}$ and $1.76 \pm 0.17 \mathrm{mg} / \mathrm{L}$, respectively. Milk produced in the organic system exhibited $7.08 \mathrm{mg} / \mathrm{L}$ nitrate and $1.61 \mathrm{mg} / \mathrm{L}$ nitrite, while milk produced in the conventional system exhibited 6.36 and $1.87 \mathrm{mg} / \mathrm{L}$, respectively. Nitrate and nitrite levels in raw milk were not affected by the production system, the period of collection, milking shift or the site of production (farm).

Keywords: raw milk; conventional system of production; organic system of production; contaminants.

\section{1 - INTRODUÇÃO}

A exposição contínua do homem a nitratos $\left(\mathrm{NO}_{3}^{-}\right)$e nitritos $\left(\mathrm{NO}_{i}^{-}\right)$é preocupante sob o ponto de vista toxicológico, devido à possibilidade de formação de compostos N-nitrosos (nitrosaminas), indutores do câncer [12, 26, 37]. No trato gastrintestinal, o nitrato pode ser convertido em nitrito pela ação de bactérias redutoras e este pode ser transformado em nitrosaminas no estômago [25]. A redução de nitratos a nitritos também pode ocorrer no próprio alimento, dependendo das condições que o mesmo oferece.

Além do risco de formação de nitrosaminas, a exposição a nitratos tem sido associada à síndrome da morte infantil súbita [15]. Niveis altos de nitrato nos alimentos ou na água de bebida prejudicam o transporte de oxigênio no sangue, especialmente em crianças [4], devido à

Recebido para publicação em 30/12/2003. Aceito para publicação em 04/05/2005(001271).

Programa de Pós-Graduação em Ciência e Tecnologia de Alimentos, CCR, UFSM.

Programa de Pós-Graduação em Zootecnia, CCR, UFSM.

Depto. de Zootecnia, CCR, Universidade Federal de Santa Maria. Email: clairo@mail.ufsm.br

Depto. de Morfologia, CCS, Universidade Federal de Santa Maria. Email:ijoni@mail.ufsm.br

Depto. de Tecnologia e Ciência de Alimentos, CCR, Universidade Federal de Santa Maria (UFSM), Camobi. CEP: 97105-900, Santa Maria-RS.E-mail:tati@ccr.ufsm.br

A quem a correspondência deve ser enviada. metemeglobinemia. Crianças com menos de 6 meses de vida são mais sensíveis à metemeglobinemia, que pode levar à anoxia e morte [21]. Os casos de intoxicação estão geralmente relacionados com a ingestão de água conten-

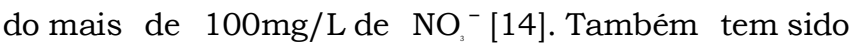
observado prejuízo na função da tireóide, decréscimo na alimentação e interferência no metabolismo das vitaminas A e E [9].

Nitratos e nitritos podem estar presentes naturalmente, nos alimentos de origem vegetal e animal e na água, em decorrência do uso de fertilizantes na agricultura [25, 31]. As principais fontes de nitrogênio residual presentes no solo são oriundas da aplicação de fertilizantes químicos e dos dejetos de animais domésticos [27]. Há estudos que comprovam a estreita relação entre o aumento de nitrato na água, em conseqüência da elevação dos níveis de nitrogênio no ambiente [35]. Assim, nitratos e nitritos podem aparecer como contaminantes do leite, embora em concentrações pouco elevadas. Os nitratos passam do solo para o pasto usado na alimentação bovina e, após a absorção pelos animais, são excretados pelo leite. No leite cru, os teores de nitrato e nitrito podem ser influenciados pelo local de origem da amostra e pela estação do ano [40]. Essa informação é confirmada em pesquisas com animais alimentados com volumosos produzidos com elevadas doses de fertilizantes nitrogenados. LEONHARD-KLUTZ, BIELAK \& ZYWCZOK [24], testando níveis crescentes de adubação nitrogenada em pas- 
tagens $(240,480$ e $720 \mathrm{~kg} / \mathrm{ha})$, em intervalos de 4 a 5 semanas, verificaram aumento nos níveis de nitrato no leite. O efeito foi menor com vacas suplementadas com nitrato. PAGE et al. [28], ao induzirem toxicose crônica de nitrato em vacas no período de lactação, via ingestão de altos $(1.600 \mathrm{mg} / \mathrm{kg}$ no concentrado e $4.000 \mathrm{mg} / \mathrm{kg}$ no feno) e baixos níveis $(356 \mathrm{mg} / \mathrm{kg}$ no concentrado e $400 \mathrm{mg} / \mathrm{kg}$ no feno), verificaram que os animais não foram afetados na quantidade de leite produzida, teor de gordura ou de nitrato no leite.

No Brasil, a produção de alimentos orgânicos é regulamentada pela Instrução Normativa $\mathrm{n}^{\mathrm{o}} 7$, de 17 de maio de 1999 [8], que relata os procedimentos que disciplinam a produção, tipificação, processamento, envase, distribuição e certificação da qualidade de produtos de produção orgânica, biodinâmica, biológica e agroecológica. De acordo com COMERÓN \& ANDREO [10], o sistema orgânico de produção é aquele sustentável, que mediante o manejo racional dos recursos naturais, e sem utilização de produtos de sínteses químicas, proporcione alimentos saudáveis. Este sistema mantém o incremento da fertilidade do solo e a biodiversidade, permitindo aos consumidores a identificação clara de características asseguradas a partir de um sistema de certificação. Segundo AROEIRA \& FERNANDES [1], a completa conversão do sistema, normalmente leva de um a três anos e durante este período o produto ainda é considerado convencional.

Alguns estudos indicam que vegetais (batata, cenoura, couve-flor, alface e outros) produzidos em sistemas convencionais apresentam conteúdo de nitrato superior ao de vegetais produzidos em sistemas biodinâmicos ou orgânicos [6]. RUTKOWSKA [32] também encontrou níveis significativamente menores de nitrato em batatas de fazendas ecológicas comparados aos níveis encontrados em fazendas convencionais, os quais foram duas vezes maiores. Já o conteúdo de nitrito não apresentou diferença entre os alimentos produzidos nas diferentes fazendas. Por outro lado, foram encontrados poucos estudos avaliando se o sistema de produção orgânica também reduz a presença de contaminantes inorgânicos em produtos de origem animal [16].

No presente trabalho, investigou-se os niveis de nitrato e nitrito em amostras de leite in natura produzido por rebanhos da Região da Depressão Central do Rio Grande do Sul, Brasil, avaliando a influência do sistema de produção empregado (convencional ou orgânico), da estação do ano, do turno da ordenha, do local de origem das amostras (propriedade produtora) e do tipo de ordenha realizada (manual ou mecânica) sobre esses niveis.

\section{2 - MATERIAL E MÉTODOS}

As amostras de leite bovino in natura foram coletadas de oito propriedades envolvidas na cadeia produtiva de leite na Região da Depressão Central do Rio Grande do Sul. Três delas foram classificadas como orgânicas de acordo com a Normativa $n^{\circ} 7$ do Ministério da Agricultura e Abastecimento [8], e as outras cinco, como convencionais. Deste total, três ordenhavam mecanicamente seus animais (uma do sistema orgânico, duas do convencional). Em cada propriedade foram coletadas amostras de leite de todos os animais, nos dois turnos (manhã e tarde), em dois dias consecutivos. As amostras da mesma propriedade foram reunidas para originar uma amostra composta, representativa, por turno de ordenha (manhã ou tarde). As coletas foram realizadas em duas estações: no inverno (julho de 2001) e no verão (março de 2002). Após a coleta, as amostras foram congeladas a $20^{\circ} \mathrm{C}$ até o momento das análises. A desproteinização das amostras foi realizada conforme descrito por CORTAS \& WAKID [11], utilizando $\mathrm{ZnSO}$ e $\mathrm{NaOH}$. Após, as amostras foram centrifugadas a $3000 \mathrm{x}$ g e o sobrenadante foi utilizado nos ensaios. O nitrito foi determinado espectrofotometricamente a $474 \mathrm{~nm}$ após diazotação com ácido sulfanílico e alfa-naftol [20]. O nitrato foi determinado após redução quantitativa a nitrito, utilizando-se grânulos de cádmio revestidos com cobre [11]. Os sobrenadantes das amostras que continham níveis de nitrato e nitrito não detectáveis pela técnica foram concentrados até 3 vezes, por evaporação em chapa de aquecimento, para aumentar a sensibilidade do ensaio.

As influências da estação do ano (inverno ou verão), do tipo de ordenha realizada (manual ou mecânica) e do local de origem das amostras (8 propriedades) foram avaliadas, separadamente, utilizando, respectivamente, teste $t$ nos dois primeiros casos e análise de variância (ANOVA) de uma via no terceiro caso. Como estes fatores não afetaram os níveis de nitrato ou nitrito das amostras, os dados foram agrupados levando em consideração apenas o sistema de produção e o turno de ordenha, e submetidos a ANOVA de duas vias $(2$ sistemas de produção $\mathrm{x}$ 2 turnos de ordenha). Optou-se por não analisar conjuntamente o efeito dos cinco fatores, pois isto reduziria muito o número de amostras em alguns grupos. As análises foram realizadas utilizando o programa Statistica 4.5.

\section{3 - RESULTADOS E DISCUSSÃO}

A concentração média encontrada nas amostras de leite foi de $6,65 \mathrm{mg} / \mathrm{L}$ de nitrato (Tabela 1) e 1,76mg/L de nitrito (Tabela 2). KORÉNEKOVÁ, KOTTEFEROVÁ \& KORÉNEK [23] detectaram 0,9mg/L de nitrato e $0,2 \mathrm{mg} / \mathrm{L}$ de nitrito em leite in natura produzido na Eslováquia, valores inferiores aos do presente trabalho. BINTORO, CANTIN-ESNAULT \& ALARU [3] detectaram entre 1,0 e $2,6 \mathrm{mg} / \mathrm{L}$ de nitrato em amostras de leite fresco da Indonésia. As amostras de leite in natura investigadas por PRZYBYLOWSKI et al. [30] na Polônia, continham nitratos em quantidades que não excediam $2 \mathrm{mg} / \mathrm{L}$ e nitritos em traços ou não detectados. Segundo WALKER [36], os teores de nitrato raramente são maiores que $5 \mathrm{mg} / \mathrm{L}$. Entretanto, níveis de nitrato entre 2$55,7 \mathrm{mg} / \mathrm{L}$ foram encontrados em amostras de leite analisadas por TSYGAMENKO et al. [34] na Ucrânia, sendo que $12 \%$ das amostras continham niveis elevados deste composto. No Brasil, segundo o RIISPOA [7], é considera- 
do impróprio para o consumo, o leite que revele a presença de nitratos ou nitritos. Assim, nenhuma das amostras analisadas no presente estudo estaria própria para o consumo, segundo esta regulamentação. SERAPHIM, SIQUEIRA \& FERNÍCOLA [33] encontraram um resultado semelhante ao do presente estudo quando analisaram amostras de leite in natura produzido na região de Alfenas, no estado de Minas Gerais $(0,02-2,1 \mathrm{mg} / \mathrm{L}$ de nitrato e $0,05-0,18 \mathrm{mg} / \mathrm{L}$ de nitrito). Na verdade, considerando que o nitrato está naturalmente presente na água e no solo, e que mamíferos podem absorver este composto e excretá-lo no leite [13, 22], seria bastante improvável não detectar nitrato e nitrito no leite empregando um método de análise sensivel.

TABELA 1 - Nitrato ( $\mathrm{mg} / \mathrm{L}_{\mathrm{de}} \mathrm{NO}_{3}$ ) em amostras de leite in natura segundo o sistema de produção e o turno de ordenha, coletadas entre 2001 e 2002 em propriedades da região da Depressão Central, RS

\begin{tabular}{cccc}
\hline \multirow{2}{*}{ Sistema de Produção } & \multicolumn{2}{c}{ Turno de Ordenha } & \multirow{2}{*}{ Média } \\
\cline { 2 - 3 } & Manhã & Tarde & $6,36 \pm 1,16$ \\
Convencional & $8,12 \pm 1,77$ & $4,59 \pm 1,40$ & \\
Orgânico & $(13)$ & $(13)$ & $7,08 \pm 1,22$ \\
$(\mathbf{n})$ & $7,31 \pm 2,11$ & $6,86 \pm 1,53$ & \\
\hline Média & $(8)$ & $(11)$ & $6,65 \pm 0,84$ \\
\hline $\begin{array}{l}\text { Os teores de nitrato são apresentados como média } \pm \text { erro padrão. } \\
n=\text { amostras representativas/rebanho. }\end{array}$
\end{tabular}

TABELA 2 - Nitrito ( $\mathrm{mg} / \mathrm{L}_{\text {de }} \mathrm{NO}_{2}^{-}$) em amostras de leite in natura segundo o sistema de produção e o turno de ordenha, coletadas entre 2001 e 2002 em propriedades da região da Depressão Central, RS

\begin{tabular}{cccc}
\hline \multirow{2}{*}{ Sistema de Produçãoo } & \multicolumn{2}{c}{ Turno de Ordenha } & \multirow{2}{*}{ Média } \\
\cline { 2 - 3 } & Manhã & Tarde & $1,87 \pm 0,23$ \\
\hline Convencional & $1,42 \pm 0,31$ & $2,33 \pm 0,33$ & \\
(n) & $(13)$ & $(13)$ & $1,61 \pm 0,23$ \\
Orgânico & $1,72 \pm 0,27$ & $1,53 \pm 0,36$ & \\
(n) & $(8)$ & $(11)$ & $1,76 \pm 0,17$ \\
\hline Média & $1,53 \pm 0,21$ & $1,96 \pm 0,25$ & \\
\hline
\end{tabular}

Os teores de nitrito são apresentados como média \pm erro padrão.

$n=$ amostras representativas/rebanho

De acordo com PEICHEVSKII \& MIKHAILOVA [29], os teores de nitrato e nitrito variam de acordo com as diferentes estações do ano, com valores geralmente maiores no período primavera/verão e menores no período outono/inverno. No entanto, no presente estudo não foram encontradas diferenças estatisticamente significativas entre as amostras coletadas no inverno e no verão, tanto nos niveis de nitrato $(7,30 \pm 1,07$ versus $5,04 \pm 1,17)$ quanto nos niveis de nitrito $(1,82 \pm 0,22$ versus $1,62 \pm 0,16)$.

A Figura 1 mostra os níveis de nitrato e nitrito de todas as amostras coletadas nas 8 propriedades rurais envolvidas no estudo. Os niveis desses compostos não foram significativamente influenciados pelo local de origem das amostras.
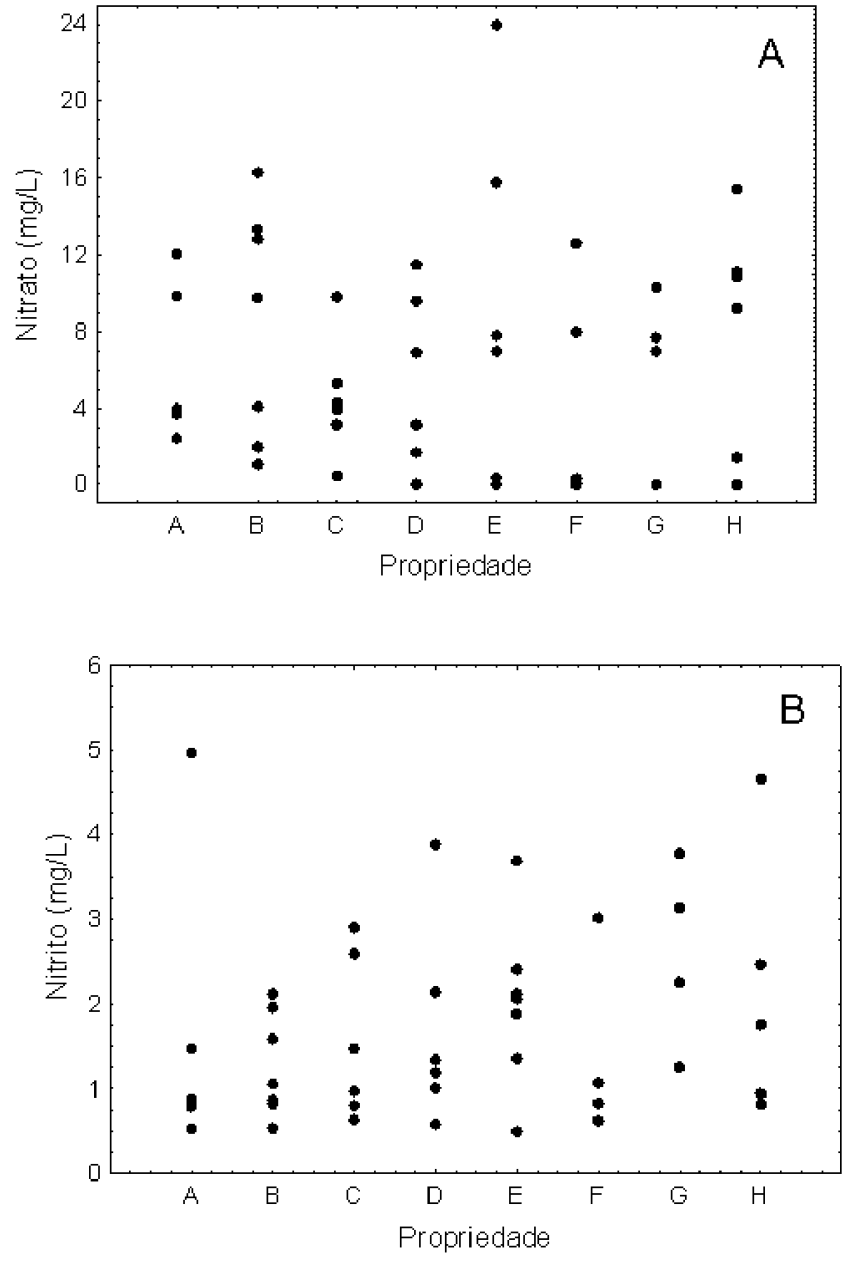

FIGURA 1 - Distribuição dos níveis de nitrato (A) e nitrito (B) nas amostras de leite in natura, segundo o local de origem das amostras (propriedade). As propriedades A, D, E, F e G são convencionais e as propriedades $\mathrm{B}, \mathrm{C}$ e H são orgânicas

As Tabelas 1 e 2 apresentam os niveis de nitrato e nitrito encontrados no leite, segundo o turno de ordenha e o sistema de produção. As concentrações de nitrato e nitrito das amostras de leite não foram influenciadas significativamente pelo turno de ordenha (Tabelas 1 e 2).

BORIN, GIUPPONI \& MORARI [5] observaram maiores niveis de nitrato na água de propriedades que utilizavam sistema convencional, quando comparadas àquelas com sistema orgânico. Segundo LEVALLOIS \& PHANEUF [25], a contaminação da água por nitrato é freqüente, devido ao uso de fertilizantes químicos na agricultura. Por outro lado, tem sido demonstrado que práticas de fertilização orgânica produzem colheitas com níveis mais baixos de nitrato, quando comparados aos das colheitas convencionais [39]. Também foram observados um melhor crescimento e reprodução de animais alimentados com produtos orgânicos [39]. No entanto, segundo WILLIAMS [38], poucos estudos têm avaliado os efeitos 
do manejo orgânico ou convencional sobre a qualidade de produtos de origem animal (carne, leite e produtos lácteos). BECK [2] observou que o leite produzido no sistema orgânico apresenta menores teores de proteína e gordura que o produzido no sistema convencional. Por outro lado, as vacas inseridas no sistema orgânico apresentaram produção mais estável, e o leite apresentou teor mais elevado de lactose e menor contagem de células somáticas [2].

No presente estudo, no entanto, não foram observadas diferenças significativas nos niveis de nitrato e nitrito encontrados nas amostras de leite coletadas de animais tratados com manejo convencional ou orgânico ( $\mathrm{Ta}$ belas $1 \quad e$ 2). GUINOT-THOMAS, JONDREVILLE \& LAURENT [16], em estudo que avaliou o efeito do sistema de produção sobre os níveis de nitrato do leite, observaram niveis maiores nas amostras do sistema orgânico, o que foi atribuído à contaminação por agentes usados na limpeza de equipamentos. No presente estudo, o tipo de ordenha (mecânica ou manual) não influenciou os níveis de nitrato $(7,48 \pm 1,33$ versus $6,10 \pm 1,09)$ ou nitrito das amostras de leite $(1,94 \pm 0,27$ versus $1,64 \pm 0,22)$. Ressalta-se que os modelos dos equipamentos de ordenha mecânica utilizados nas propriedades estudadas são do tipo balde-ao-pé, nos quais há maior simplicidade tanto na lavagem quanto no uso de detergentes.

HAMBITZER et al. [17] observaram uma correlação entre os níveis plasmáticos de nitrato e a ingestão de nitrato em ruminantes adultos. No entanto, de acordo com HAPKE [18] e HÜSLER \& BLUM [19], o efeito da ingestão de nitrato ou nitrito sobre suas concentrações endógenas é muito maior nos animais jovens do que no gado com o pré-estômago funcionante, onde microrganismos metabolizam primariamente o nitrato a nitrito, e depois a hidroxilamina e amônia.

KAMMERER, PINAULT \& POULIQUEN [22] observaram que a adição de até $180 \mathrm{mg} / \mathrm{L}$ de nitrato à água fornecida a vacas leiteiras de uma fazenda não afetou significativamente o conteúdo de nitrato no leite. Da mesma forma, DUSDIEKER et al. [13] observaram que a adição de $100 \mathrm{mg} / \mathrm{L}$ de nitrato na água consumida por mulheres em fase de lactação não elevou as concentrações de nitrato no leite das lactantes. Os resultados de ambos os estudos sugerem que o conteúdo de nitrato e nitrito no leite é pouco sensivel a flutuações nos niveis desses compostos na dieta dos animais lactantes. Os resultados obtidos no presente estudo corroboram a hipótese apresentada acima, pois revelam que a produção orgânica não foi capaz de reduzir os níveis destes compostos no leite, ainda que diversos trabalhos anteriores tenham demonstrado uma redução destes contaminantes em vegetais [32, 39]. Assim, parecem ser necessárias grandes alterações nos niveis de nitrato e nitrito da dieta, como aquelas impostas por LEONHARD-KLUTZ, BIELAK \& ZYWCZOK [24], para que ocorram variações nos níveis destas substâncias no leite.

Independentemente do sistema de produção, os valo- res médios de nitrato e nitrito encontrados no leite produzido em propriedades da Região da Depressão Central do Estado do Rio Grande do Sul são geralmente mais elevados do que aqueles referidos em pesquisas conduzidas em outros países.

\section{4 - CONCLUSÕES}

Os resultados do presente estudo, juntamente com outros trabalhos que revelaram a presença de nitrato e/ou nitritos em leite produzido no Brasil e em outros países, apontam para a necessidade de uma revisão da legislação brasileira, que considera impróprio para o consumo o leite que revele a presença desses contaminantes. Considerando que a maioria das amostras de leite analisadas neste trabalho e em outros realizados no Brasil e no exterior $[33,36]$ apresentam concentrações de nitrato abaixo de $10 \mathrm{mg} / \mathrm{L}$, e que este é o limite para a presença de nitratos em água potável [12], sugere-se que poderia ser estabelecido como limite para a presença natural de nitrato no leite um valor próximo a esse.

Os níveis de nitrato e nitrito encontrados nas amostras de leite in natura produzidos na região da Depressão Central do Rio Grande do Sul são relativamente elevados quando comparados aos níveis encontrados em amostras de leite produzido em outras regiões do país e do mundo. Além disso, os níveis de nitrato e nitrito presentes nas amostras de leite não foram influenciados nem pelo sistema de produção, nem por fatores como estação do ano, turno de ordenha, local de origem das amostras ou tipo de ordenha realizada.

\section{5 - REFERÊNCIAS BIBLIOGRÁFICAS}

[1] AROEIRA, L.J.M.; FERNANDES, E.N. Produção orgânica de leite: um desafio atual. Informe agropecuário, v.22, n.211, p.53-57, 2001.

[2] BECK, L.I. Análise e Comparação de Sistemas Convencional e Agroecológico de produção de leite. Santa Maria, 2002. 95p. Dissertação (Mestre em Zootecnia), Universidade Federal de Santa Maria, 2002.

[3] BINTORO, V.P.; CANTIN-ESNAULT, D.; ALARU, J. A survey of nitrate contents in Indonesian milk by enzymic analysis. Food Additives and Contaminants, v. 13, n.1, p.77-87, 1996.

[4] BOER, I. J. M. Environmental impact assessment of conventional and organic milk production. Livestock Production Science, v. 80, p. 69-77, 2003.

[5] BORIN M., GIUPPONI C., MORARI, F. Effects of four cultivation systems for maize on nitrogen leaching. 1. Field experiment. European Journal of Agronomy, v.6, n.1-2, p.101-112, 1997.

[6] BOURN, D.; PRESCOTT, J. A comparison of the nutritional value, sensory qualities, and food safety of organically and conventionally produced foods. Critical Reviews in Food Science and Nutrition, v.42, n.1, p.134, 2002.

[7] BRASIL. MINISTÉRIO DA AGRICULTURA. R.I.I.S.P.O.A. 1980. Regulamento da Inspeção Industrial e Sanitária de 
Produtos de Origem Animal (Aprovado pelo decreto $\mathrm{n}^{\mathrm{o}}$ 30690 , de 20.03.52, alterado pelo decreto $\mathrm{n}^{\mathbf{0}} 1255$, de 25.06.52). Brasília. 66p.

[8] BRASIL. MINISTÉRIO DA AGRICULTURA E DO ABASTECIMENTO. Instrução Normativa № 7, de 17 de maio de 1999. Dispõe sobre normas para a produção de produtos orgânicos vegetais e animais. Diário Oficial da República Federativa do Brasil, Brasília, v. 94, p. 1114, 19 de maio de 1999. Seção 1.

[9] BRUNING-FANN, C.S.; KANEENE, J.B. The effects of nitrate, nitrite, and $\mathrm{N}$-nitroso compounds on animal health. Veterinary and Human Toxicology, v.35, n.3, p.237-253, 1993.

[10] COMERÓN, E.A.; ANDREO, N.A. Produção, industrialização e comercialização do leite orgânico na Argentina. In: SIMPóSIO SOBRE SUSTENTABILIDADE DA PECUáRIA LEITEIRA NO BRASIL, 2, 2000, Goiânia. Anais... Tecnologias utilizadas pelos produtores de leite de Goiás e suas relações com questões de sustentabilidade e competitividade do segmento da produção. Juiz de Fora: EMBRAPA Gado de Leite, 2000.

[11] CORTAS, N. K.; WAKID, N. W., Determination of inorganic nitrate in serum and urine by a kinetic cadmium reduction method. Clinical Chemistry, v.36, n.8, p. 1440-1443, 1990.

[12] DUARTE, M.; MÍDIO, A.F. Nitratos e nitritos em alimentos. Cadernos de Nutrição, v. 12, p.19-30, 1996.

[13] DUSDIEKER, L.B., STUMBO, P.J.; KROSS, B.C.; DUNGY, C.I. Does increased nitrate ingestion elevate nitrate levels in human milk? Archives of Pediatrics and Adolescent Medicine, v.150, n.3, p.311-314, 1996.

[14] FAN, A.M., WILHITE, C.C., BOOK, S.A. Evaluation of the nitrate drinking water standard with reference to infant methemoglobinemia and potential reproductive toxicity. Regulatory Toxicology and Pharmacology, v. 7, p. 135148, 1987.

[15] GEORGE, M., WIKLUND, L., AASTRUP, M., POUSETTE, J., THUNHOLM, B., SALDEEN, T., WERNROTH, L., ZAREN, B., HOLMBERG, L. Incidence and geographical distribution of sudden infant death syndrome in relation to content of nitrate in drinking water and groundwater levels. European Journal of Clinical Investigation, v. 31, p. 1083-1094, 2001.

[16] GUINOT-THOMAS, P., JONDREVILLE, C., LAURENT, F. Comparison of milk from farms with biological, conventional and transitional feeding. Milchwissenschaft, v. 46, p. 779-782, 1991.

[17] HAMBiTZER, R., VELKE, H., RAMM, K., SOMMER, H. Bewertung der Nitratbelastung von laktierenden und trockenstehenden Milchkühen anhand der nitratwerte in Blutplasma. Tierärztliche Umschau, v.42, p.775-780, 1987.

[18] HAPKE, H.J. Toxikologie für Veterinärmediziner, 2 edition. Stuttgart: VERLAG F. ENKE, 1998.

[19] HUSLER, B.R., BLUM, J.W. Blood plasma response and urinary excretion of nitrite and nitrate in milk-fed calves after oral nitrite and nitrate administration. Journal of Nutritional Biochemistry, v.12, p.304-309, 2001.

[20] INSTITUTO ADOLFO LUTZ. Normas Analíticas do
Instituto Adolfo Lutz. São Paulo, 1985. p.94-102.

[21] JOHNSON, C.J., BONRUD, P.A., DOSCH, T.L., KILNESS, A.W., SENGER, K.A., BUSCH, D.C., MEYER, M.R. Fatal outcomes of methemoglobinemia in an infant. JAMA: The Journal of The American Medical Association, v. 257, p. 2796-2797, 1987.

[22] KAMMERER, M.; PINAULT, L.; POULIQUEN, H. Content of nitrate in milk. Relationship with its concentration in the water supply for livestock. Annales de Recherches Veterinaires, v.23, n.2, p.131-138, 1992.

[23] KORÉNEKOVÁ, B.; KOTTFEROVÁ, J.; KORÉNEK, M. The fat of added nitrate used in the manufacture of Emmental cheese. Food Additives and Contaminants, v.17, n.5, p.373-377, 2000.

[24] LEONHARD-KLUTZ, I.; BIELAK, F.; ZYWCZOK, H. Composition and properties of milk from cows fed green forage cultivated with intensive nitrogen fertilization. In: INTERNATIONAL DAIRY CONGRESS, 20, 1978, Krakov, Poland. Proceedings... Krakov: Inst. of Zootech, 1978. p.74-75.

[25] LEVALLOIS, P., PHANEUF, D. Contamination of drinking water by nitrates: analysis of health risks. Canadian Journal of Public Health, v.85, n.3, p.192-196, 1994.

[26] LIJINSKI, W. Induction of tumours in rats by feeding nitrosatable amines together with sodium nitrite. Food and Chemical Toxicology, v. 22, p. 715-720, 1984.

[27] OENEMA, O.; BOERS, P. C. M.; VAN EERDT, M. M.; FRATERS, B.; VAN DER MEER, H. G.; ROEST, C. W. J.; SCHRÖDER, J. J.; WILLEMS, W. J. Leaching from agriculture to groundwater: the effect of policies and measures in the Netherlands. Environmental Pollution, v. 102, p. 471-478, 1998.

[28] PAGE, R. D.; GILSON W. D.; GUTHRIE L. D.; MERTENS D. R.; HATCH R. C. Serum progesterone and milk production and composition in dairy cows fed two concentrations of nitrate. Veterinary and Human Toxicology, v.32, n.1, p.27-31, 1990.

[29] PEICHEVSKII, I. \& MIKHAILOVA, G. Contents of nitrate and nitrite in milk in relation to region and season. Khranitelna-Promishlenost, v.41, n.5-6, p.26-27, 1992.

[30] PRZYBYLOWSKI, P., KISZA, J., JANICKA, B., SAJKO, W. Presence of nitrates and nitrites in raw milk as subject to the system of milk purchasing. Rocziniki Panstwowego Zakladu Higieny, v.40, n.1, p.6-15, 1989.

[31] RATH, S., XIMENES, M.I.N., REYES, F.G.R. Nitrate and nitrite in vegetables cultivated at Distrito Federal: preliminary study. Revista do Instituto Adolfo Lutz, v. 54, p. 126-130, 1994.

[32] RUTKOWSKA, B. Nitrate and nitrite content in potatoes from ecological and conventional farms. Rocziniki Panstwowego Zakladu Higieny, v.52, n.3, p.231-236, 2001.

[33] SERAPHIM, K.R., SIQUEIRA, M.E.P.B., FERNÍCOLA, N.A.G. Nitrates and nitrites in raw and commercially pasteurized milk in the Ciudad Alfenas region, Minas Gerais, Brazil. Alimentaria, v. 296, p. 49-52, 1998.

[34] TSYGAMENKO, O.I., EMCHENKO, N.L., LAPCHENKO, V.S., TSYPKO, M.I., MIKHALLUK, E.N., STAKHURSKAIA, 
L.V., STADNICHUK, N.A., VEZIKA, S.S. Nitrates in cow's milk and in various milk products in the Ukraine. Voprosy Pitaniia, n.3, p.45-49, 1991.

[39] WANG, S. J.; FOX, D. G.; CHERNEY, D. J. R.; KLAUSNER, S. D.; BOULDIN, D. R. Impact of Dairy Farming on Well Water Nitrate Level and Soil Content of Phosphorus and Potassium. Journal of Dairy Science, v.82, n.10, p.2164-2469, 1999.

[36] WALKER, R. Nitrates, nitrites and n-nitrosocompunds: a review of the ocurrence in food and diet and the toxicological implications. Food Additives and Contaminants, v.6, n.7, p.717-768, 1990.

[37] WHO. WORLD HEALTH ORGANIZATION. Nitrates, nitrites and N-nitroso compounds. Genebra, 1978. 107. [Environmental Health Criteria, n. 5].

[38] WILLIAMS, C.M. Nutritional quality of organic food: shades of grey or shades of green? Proceedings of the Nutrition Society, v. 61, p. 19-24, 2002.
[39] WORTHINGTON, V. Effect of agricultural methods on nutricional quality: A comparison of organic with convencional crops. Alternative therapies in health and medicine, v. 4, n. 1, 58, 1998.

[40] ZBIKOWSKI, Z; ZBIKOWSKA, A.; BARANOWSKA, M. Content of nitrates and nitrites in raw milk in different regions of the country. Roczniki Panstwowego Zakladu Higieny, v. 51, n.1, p. 29-35, 2000.

\section{6 - AGRADECIMENTOS}

Os autores agradecem o auxilio de Simone Bertazzo Rossato, aluna do Programa de Pós-Graduação em Ciência e Tecnologia dos Alimentos-CCR-UFSM, na realização de algumas das análises de nitrato e nitrito. I. Costabeber é bolsista PROFIX do CNPq Brasil e T. Emanuelli é bolsista pesquisadora do CNPq. 